Molecules 2000, 5, 1011-1013

\title{
molecules
}

ISSN 1420-3049

(C) 2000 by MDPI

http://www.mdpi.org

\section{2-Substituted-1,3,2-dithioborolans as Chiral Lewis Acid Catalysts}

\section{Joshua Howarth* and Paul Glynn}

School of Chemical Sciences, Dublin City University, Glasnevin, Dublin 9, Ireland

Tel.: +353 1 7045312, Fax: +353 1 7045503, E-mail: howarthj@ccmail.dcu.ie

Received: 29 June 2000 / Accepted: 10 August 2000 / Published: 15 August 2000

\begin{abstract}
Dithioborolans substituted at the 2-position with various homochiral groups can act as chiral Lewis acids in the Diels-Alder reaction between crotonaldehyde or methacrolein with cyclopentadiene. The endo:exo selectivities obtained were good although the enantiomeric excesses were low to moderate.
\end{abstract}

Keywords: boron heterocycle, Lewis acid, Diels-Alder.

\section{Introduction}

In a previous publication [1] we showed that 2-chloro-1,3,2-dithioborolan (1) was a Lewis acid. In a follow up investigation we have looked at whether substitution of the chlorine for a homochiral group RX (Scheme 1) would result in a homochiral Lewis acid which could produce enantiomeric excesses in the representative Diels-Alder reaction between crotonaldehyde (2) or methacrolein (3) with cyclopentadiene (4) (Scheme 2). Interest in the homochiral Lewis acids for asymmetric control of the Diels-Alder reaction has been enormous [2]. This investigation has tried to assess 2-substituted-1,3,2-dithioborolans for their viability as a new class of homochiral Lewis acids.

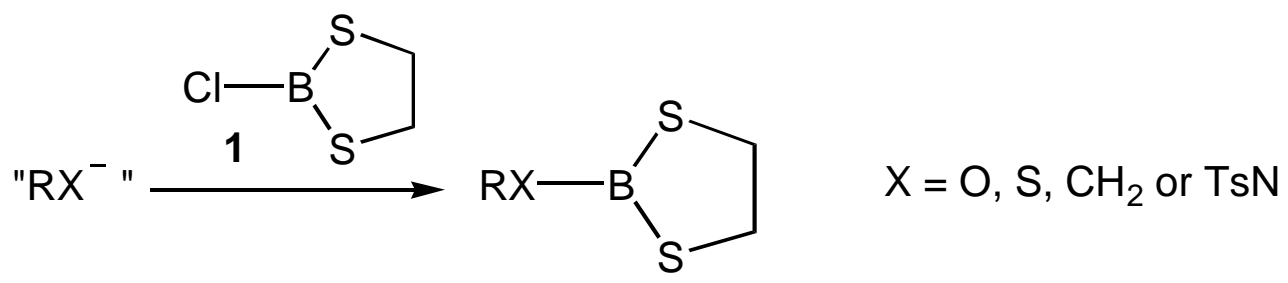

Scheme 1. 


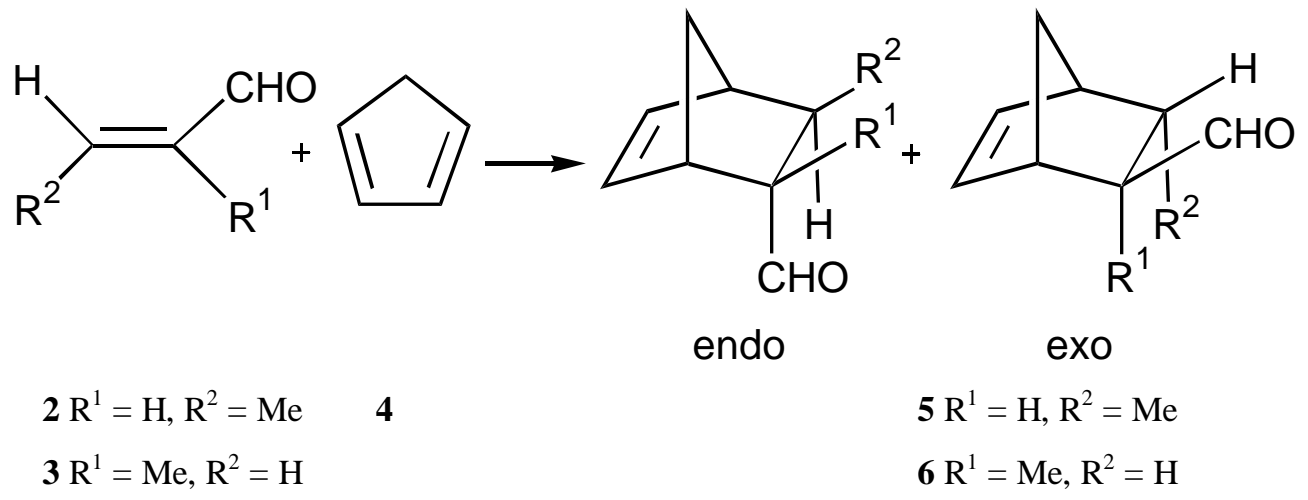

Scheme 2.

\section{Results and Discussion}

It can be seen from the endo:exo ratios in Table 1 that substitution of chlorine on $\mathbf{1}$ for sulfur, oxygen or carbon does not radically diminish the Lewis acid activity of the dithioborolan system, since these ratios remain substantially enhanced relative to the uncatalysed reaction, and are similar to those found for 2-chloro-1,3,2-dithioborolan under similar reaction conditions [1]. However, when the chlorine is substituted for nitrogen it appears that there must be an electron withdrawing group on the nitrogen for Lewis acid catalysis to be seen, as in example 9, Table 1. Similar experiments to those above were carried out using (S)-(-)-methylbenzylamine and achiral amines and no Lewis acid activity was observed.

Table 1.

\begin{tabular}{|c|c|c|c|c|c|c|c|}
\hline \multirow{2}{*}{\multicolumn{2}{|c|}{$\begin{array}{c}\text { Compound/Parent Chiral } \\
\text { Group for RX } \\
\end{array}$}} & \multicolumn{3}{|c|}{ Crotonaldehyde } & \multicolumn{3}{|c|}{ Methacrolein } \\
\hline & & Yield\% & ee\% & Endo:Exo & Yield & ee\% & Endo:Exo \\
\hline 7 & Menthol & 61 & 33 & $90: 10$ & 69 & 54 & $8: 92$ \\
\hline 8 & Neothiomenthol & 64 & 35 & $94: 6$ & 35 & 42 & 9:91 \\
\hline 9 & $\begin{array}{l}(\mathrm{S})-(-)-\mathrm{N}-\mathrm{T} \text { osyl [3] methyl- } \\
\text { benzylamine }\end{array}$ & 51 & 38 & $90: 10$ & 43 & 31 & $11: 89$ \\
\hline & $\begin{array}{l}\text { (S)-(+)-Bromo-2-methyl- } \\
\text { butane }\end{array}$ & 42 & 18 & $89: 11$ & 34 & 20 & $11: 89$ \\
\hline & $(\mathrm{R})-(+)-\mathrm{BINOL}$ & 25 & 41 & $90: 10$ & 30 & 56 & $11: 89$ \\
\hline & $(2 \mathrm{R}, 3 \mathrm{R})-2,3$-Butanediol & 40 & 24 & 90:10 & 70 & 31 & $8: 92$ \\
\hline
\end{tabular}

\section{Conclusions}

From molecular modeling studies we have concluded that the enantioselectivities observed are low to moderate due to the flat disposition of the dithioborolan ring. This allows the dienophile to position itself outside the control of the chiral substituent. We think that if homochiral dithiols are used to form the dithioborolan ring system an enhancement of enantioselectivities will be found. This avenue is currently being investigated. 


\section{Experimental}

The Lewis acid catalyst was made in situ by adding butyllithium (1.1 eq) to a solution of the compounds 7-9 (1.2 eq.) in THF under nitrogen, followed by addition of 1 (1.0 equivalents) [3]. In the case of $\mathbf{1 0}$ the Grignard reagent was formed and $\mathbf{1}$ added to the solution and in the case of $\mathbf{1 1}$ and $\mathbf{1 2}, 2.0$ equivalents of $\mathbf{1}$ are added to the alkoxide formed by adding 2.2 equivalents of butyllithium to 1.2 equivalents of the diol. The Lewis acid catalyst ( 0.2 eq.), cyclopentadiene (4, 5.0 eq.) and the dienophile 2 or 3 (1.0 eq.) were stirred under nitrogen at $-78^{\circ} \mathrm{C}$ in THF for $4 \mathrm{~h}$. The solvent was then removed in vacuo, and the resulting residue was distilled to give the aldehyde $\mathbf{5}$ or $\mathbf{6}$. Reaction between the dienophiles $\mathbf{2}$ and $\mathbf{3}$ and cyclopentadiene (4) did not occur without a catalyst under these conditions. The enantiomeric excess was determined by derivatisation of the resulting aldehyde 5 or $\mathbf{6}$ with (2S, 4S)-(+)pentanediol and using ${ }^{1} \mathrm{H}-\mathrm{NMR}$ or chiral capillary GC. The results of the experiments are given in Table 1.

\section{References and Notes}

1. Howarth, J.; Helmchen, G.; Kiefer, M. Tetrahedron Lett. 1993, 34, 4095.

2. Maruoka, K.; Yamamoto, H. in Catalytic Asymmetric Synthesis; Ojma, I., Ed.; VCH Publishers Inc.: 1993; Chapter 9.

3. Mikolajczyk, M.; Perlikowska, W.; Omelanczuk, J. Synthesis 1987, 1009.

Sample Availability: Not applicable.

(C) 2000 by MDPI (http://www.mdpi.org). 